\title{
Bingham Sealing and Application in Vacuum Clamping
}

\author{
S.M.Yao, Wee Kin Teo, Zunmin Geng, Sam Turner, Keith Ridgway \\ Address: Advanced Manufacture Research Centre (AMRC) with Boeing, University \\ of Sheffield, UK
}

Email: yaoshaoming@126.com

\begin{abstract}
Vacuum clamping is extensively used in shell machining. In this paper a Bingham Sealing (BS) is presented and formulized based on Bingham plastic performance. The sealing capability of BS is evaluated in various cases. A new Bingham plastic is developed and the yield stress is measured. The performances of "O"ring sealing and BS with the developed Bingham plastic are compared to the static experiment. In this experiment the same vacuum is achieved and the distortion of the blade with BS is better than that with "O" ring sealing.
\end{abstract}

\section{Introduction}

In the aeronautical and astronautical industries, aircraft and spacecraft components are commonly produced in shell form to keep their mass to a minimum. Examples of shell-like components include solid/hollow blades, leading edges, and solid/hollow vanes. When machining shell components, it is found that no position is available for clamping purposes because the whole surface (aerofoil) is to be machined. Conventionally a vacuum is applied on the back of the surface (aerofoil) to be machined as the clamping approach. However, an ' $\mathrm{O}$ ' ring for sealing has to be supplied in a closed ' $\mathrm{O}$ ' ring groove and pressed to some extend to ensure a good sealing effect. So a considerable deformation is impaled to the thin-wall component because of the vacuum pressure. Upon finish machining, the deformation would be entirely restored (released) when removed from fixture. Thus results in poor tolerance part and increased manufacturing cost.

In 1992 [1] Hoffmann summarized the rules that vacuum clamping on CNC-woodworking machines in the slip-Tilt-Polar-diagram. In 2004 Anon [2] used vacuum clamping in deburring. In 1986 Witte [3] used vacuum clamping technique in thin-walled work-piece machining.

A UK patent [4] describes an adaptive design of fixture for providing support and rigidity to thinwalled shell/cylindrical components during machining. However, many shell components (aerofoils, for example) are not cylindrical, or indeed symmetrical and would not benefit from the fixture described. An air bag is required in this patent which can not be used for support and clamping on the same surface (aerofoil).

An alternative clamping method uses wax as a clamping medium. Whereby is commonly used in the engine industries when machining the ends of shell components since it provides both clamping and damping. This clamping must be applied on both side of the aerofoil for its effectiveness. In order to conform to the profile of the component surface, the wax is heated and cast it over the desired area. This process requires heating equipment and, in some cases, large amounts of energy to cast the wax. In addition, the wax must be safely disposed of and the emission level of the volatile chemicals that result from melting the wax must be carefully controlled and monitored. 
Therefore, there is a need for a sealing technique to seal the gap produced between component and fixture during vacuum clamping in shell machining that is cost effective, environmentally friendlier and less deformation produced than current methods.

A Bingham plastic is a viscoplastic material that behaves as a rigid body at low stresses but flows as a viscous fluid at high stress. It is named after Eugene C. Bingham who proposed its mathematical form [5]. If the pressure at one end of a pipe is increased a stress is applied on the fluid as a result that initiate movement (shear stress) and the volumetric flow rate increases proportionally. However for a Bingham Plastic fluid, flow movement will only commence when a certain value of yield stress is reached. Beyond this point the flow rate increases steadily with increasing shear stress. This is as presented by Bingham via his observation of the experimental study of paints [6].

A shaft seal with an energized electro-rheological fluid (ERF) is presented [7]. 2D model established based on Bingham plastic flowing between two parallel electrode plates and it is witnessed that leakage will always exist, whenever the shaft is rotating, regardless pressure gradient along the seal annulus. The simulation is proven via experimental trials. In 2004 Wang [8] disclosed a patent of roller with a magnetic-rheological fluid (MRF) sealing. Permanent magnet is used to drive magnetic rheological fluid and achieve a sealing capability.

Both of ERF and MRF are costly to apply in machining and the electrical or magnetic loop makes sealing mechanism too complicated to integrate in a fixture with vacuum clamping.

Bingham plastic is used to grout or fill open voids in fractured rock by introducing a fluid through boreholes under pressure [9]. Jiro [10] disclosed a gas barrier film for gas sealing containing a substrate film and a barrier layer and the working process is simplified. In this patent the barrier layer is restricted in an area by an additional seal. Freyer disclosed [11] a sealing technique for a void incompletely filled with a cast material. An expandable material is used to expand when a void filled with a cast material.

A sealing mechanism based on Bingham plastic and its unique performances could be constructed if a Bingham plastic with suitable performances is available. In this paper, the basic analytic model of a Bingham sealing is established and approaches to enhance sealing capability are discussed. A method is developed to evaluate the yield strength of Bingham plastic. With this method a water-based Bingham plastic is measured and the seal design is used in a vacuum clamping based on supposed Bingham sealing and the water-based Bingham plastic. Experiments had shown that this sealing mechanism works well.

\section{Bingham Plastic}

Bingham plastic is a solid when the shear stress $(\tau)$ less than a critical value, yield stress, $\tau_{0}$. Once the shear stress exceed the yield stress the material flows in such a way that the shear rate, $\partial \mathrm{u} / \partial \mathrm{y}$, is proportional to the amount by which the applied shear stress exceeds the yield stress, as shown in Fig.1.

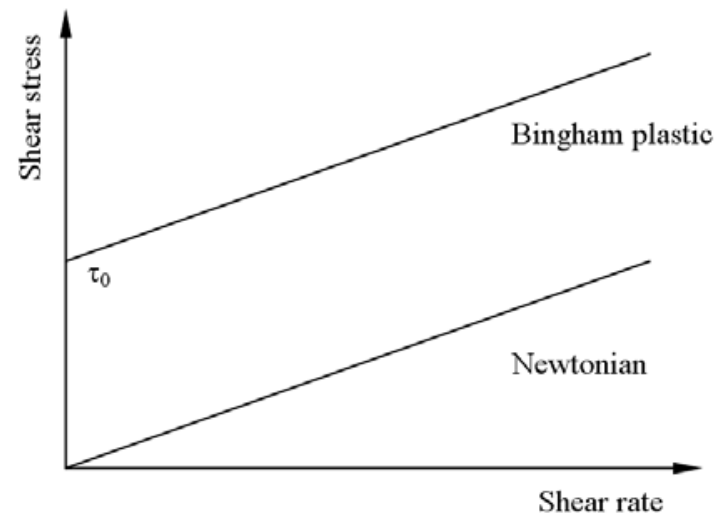

Fig.1 Bingham plastic performance

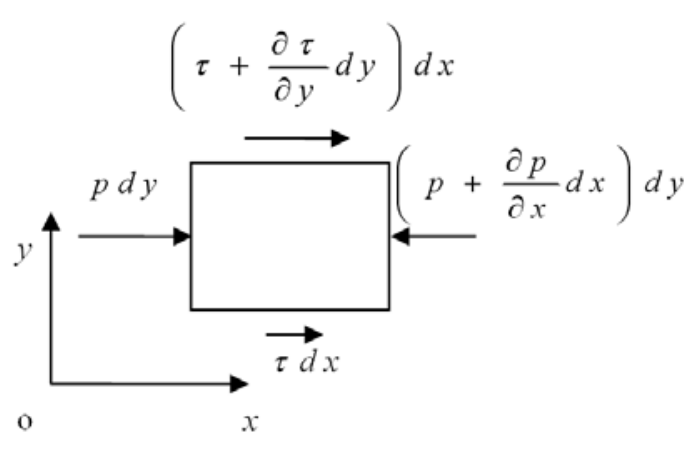

Fig.2 2D small element of Bingham plastic $\tau$ : shear stress; $p$ : pressure; $\mathrm{x}, \mathrm{y}: 2 \mathrm{D}$ coordinates 


$$
\frac{\partial u}{\partial y}= \begin{cases}0, & \tau<\tau_{0} \\ \left(\tau-\tau_{0}\right) / \mu, \tau \geq \tau_{0}\end{cases}
$$

For a small element in flow area is shown in Fig.2. Suppose pressure, p, is uniform along y direction and velocity, $\mathrm{v}$, along y direction is zero.

$$
\frac{\partial \tau}{\partial y}=\frac{\partial p}{\partial x}
$$

When shear stress exceeds the yield stress the material will flow as according to:

$$
\tau-\tau_{0}=\mu \frac{\partial u}{\partial y}
$$

\section{Bingham Sealing}

The performance of Bingham plastic can be used to achieve a sealing function.

An infinite long gap between two components is filled with Bingham plastic with curved coordinates system as shown in Fig3. Ambient pressure, $\mathrm{p}_{0}$, is at the origin, o', of the curve coordinates system and sealing pressure, $\mathrm{p}(\mathrm{s})$, on another side. Local curvature, $r(s)$, is a function of curve coordinate, $s$.

Suppose the local curvature, $\mathrm{r}(\mathrm{s})$, is considerably larger than the sealing gap thickness, $\mathrm{h}(\mathrm{s})$, and the local curvature effect is negligible. A small segment of the gap is as shown in Fig.4. Pressure, p(s), and sealing gap thickness, $\mathrm{h}(\mathrm{s})$, are function of curve coordinate, $\mathrm{s}$. When the sealing fails the yield stress acts on both sides of the sealing material and yield

$$
h(s) \frac{\partial p}{\partial s}=2 \tau_{0}
$$

The dealing pressure, $p$, is an integral of pressure difference along the curve.

$$
p-p_{0}=\int_{0}^{L} \frac{\partial p}{\partial s} d s=\int_{0}^{L} \frac{2 \tau_{0}}{h(s)} d s
$$

When the sealing gap thickness is a constant, the sealing pressure will be reduced as

$$
p=2 \tau_{0} \frac{L}{H}
$$

Where, $\mathrm{L}$ is sealing length, $\mathrm{H}$ is sealing gap thickness.

The sealing pressure is directly proportional to yield stress of Bingham plastic and the ratio of sealing length and sealing gap.

\subsection{Axisymmetrical Sealing}

Bingham sealing can be used in various engineering cases, in particular for both static and dynamic cases at low speed.

A cylindrical sealing gap with a uniform sealing thickness is as shown in Fig.5. The shaft is enclosed by two parts with half cylindrical surface and the

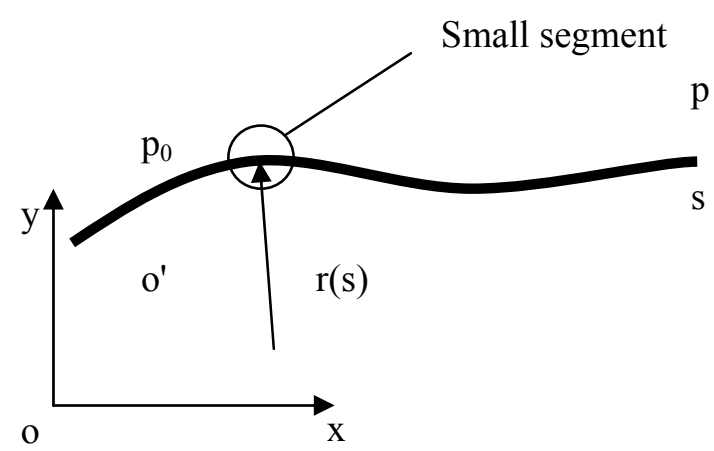

Fig.3 Section of infinite long sealing gap with Bingham plastic

s: curve coordinate; $r(s)$ : local curvature; o': origin of curve coordinate;

$\mathrm{p}_{0}$ : ambient pressure; $\mathrm{p}$ : sealing pressure

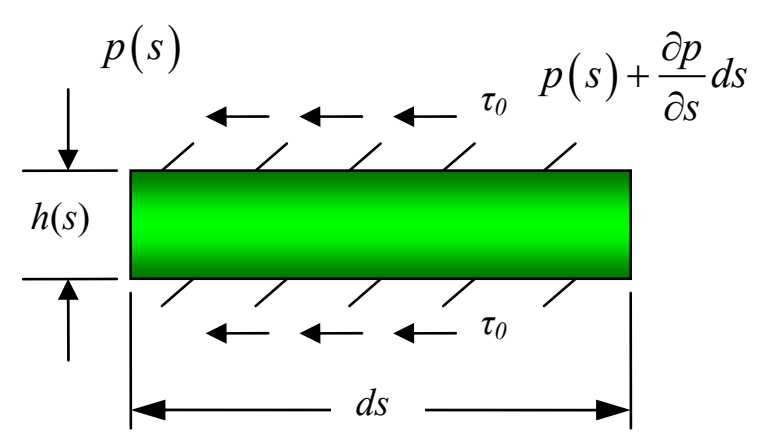

Fig.4 A segment of sealing gap $h(\mathrm{~s})$ : gap thickness; $p(\mathrm{~s})$ : pressure; $d \mathrm{~s}$ : segment length 
gap is filled with Bingham plastic. In another case shown in Fig.6 a shaft equipped sealing step is enclosed by two parts with half corresponding geometries and the gap is filled with Bingham plastic. The sealing step will increase the sealing length, L, considerably and the sealing pressure promoted.

$$
\begin{gathered}
L=l+N\left(D_{2}-D_{1}\right) \\
p-p_{0}=2 \tau_{0} \frac{l+N\left(D_{2}-D_{1}\right)}{H}
\end{gathered}
$$

Where: $l$ is width of seal, $\mathrm{N}$ is the number of sealing steps, and $D_{1}$ and $D_{2}$ are diameters of the shaft and sealing step.

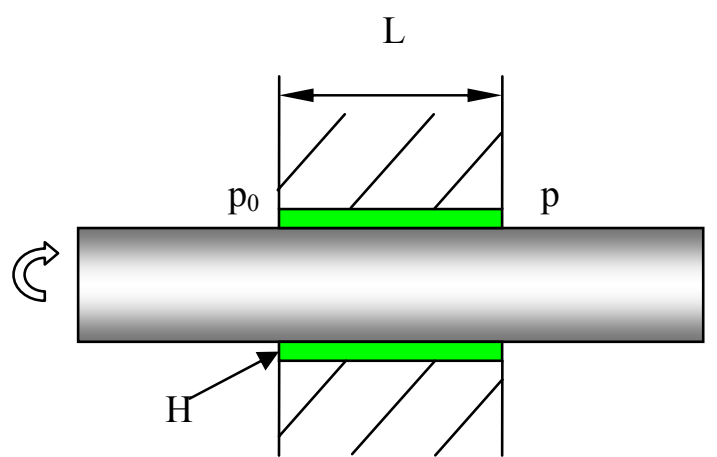

Fig.5 cylindrical sealing gap with a uniform sealing thickness

$\mathrm{p}_{0}$ : ambient pressure; $\mathrm{p}$ : sealing pressure

L: sealing length; H: sealing gap thickness

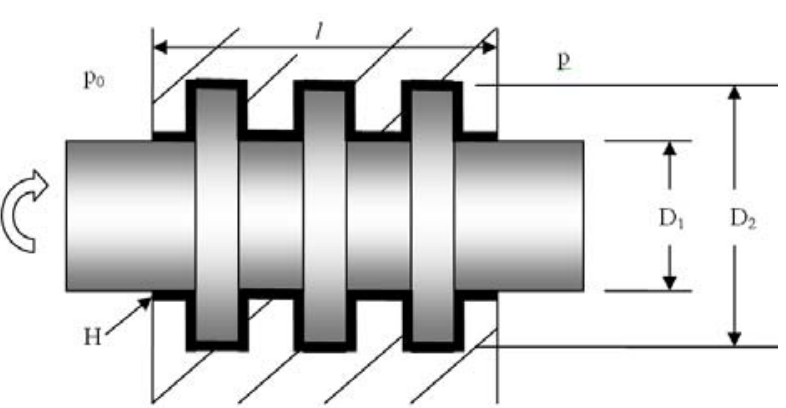

Fig.6 axisymmetrical sealing gap with sealing steps

$l$ is width of seal, $D_{1}$ and $D_{2}$ are diameters of the shaft and sealing step

\subsection{Plane-to-Plane Sealing}

The Bingham sealing can be applied in vacuum fixture for holding the part to machining facilities. This is ideal for a shell structure with a thin wall, such as blades. A basic model for vacuum clamping is as shown in Fig.7. Bingham plastic is painted with an area for which the boundaries are restricted by the fixture edge and isobaric grooves. Then a shell is mounted on to the solid fixture with Bingham sealing that serves as the method of holding when the vacuum pump is in operation.

\section{Yield Stress Measurement}

A Bingham plastic consists of the following:

$\begin{array}{cccccc}\text { Elements } & \mathrm{H}_{2} \mathrm{O} & \mathrm{SiO}_{2} & \mathrm{Al}_{2} \mathrm{O}_{3} & \mathrm{CaO} & \text { else } \\ \text { proportions } & 83 \% & 7.5 \% & 2.7 \% & 0.8 \% & 6 \%\end{array}$

The measurement system is set up, as shown in Fig.8, to testify the yield stress of this Bingham plastic. Bingham plastic, as denoted by 1, is painted on top sides of both fixed blocks and a movable plate 3 is clamped between two fixed block 2 with a gap of 0 to $0.5 \mathrm{~mm}$. The weight force is applied on the bottom of the movable plate at a gradual increase until the movable plate starts to move. The measurement results are listed in Table 1.

The yield stress is evaluated as 


$$
\tau_{0}=\frac{F}{2 \pi d^{2}}
$$

Where, $\mathrm{F}$ is total force applied including the weight of movable plate and $\mathrm{d}$ is diameter of Bingham sealing area.

Table 1 Yield stress test for Bingham plastic, $d=0.075(\mathrm{~m})$

\begin{tabular}{ccccccc}
\hline Test & 1 & 2 & 3 & 4 & 5 & average \\
\hline$F(\mathrm{~N})$ & 5.5 & 5.6 & 5.5 & 5.4 & 5.7 & 5.56 \\
$\tau_{0}(\mathrm{~Pa})$ & 622 & 634 & 622 & 611 & 645 & 626.8 \\
\hline
\end{tabular}

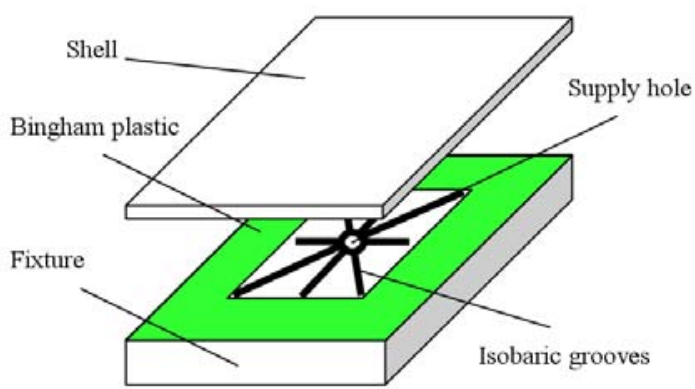

Fig.7 Bingham sealing in a vacuum clamping fixture for a thin wall shell

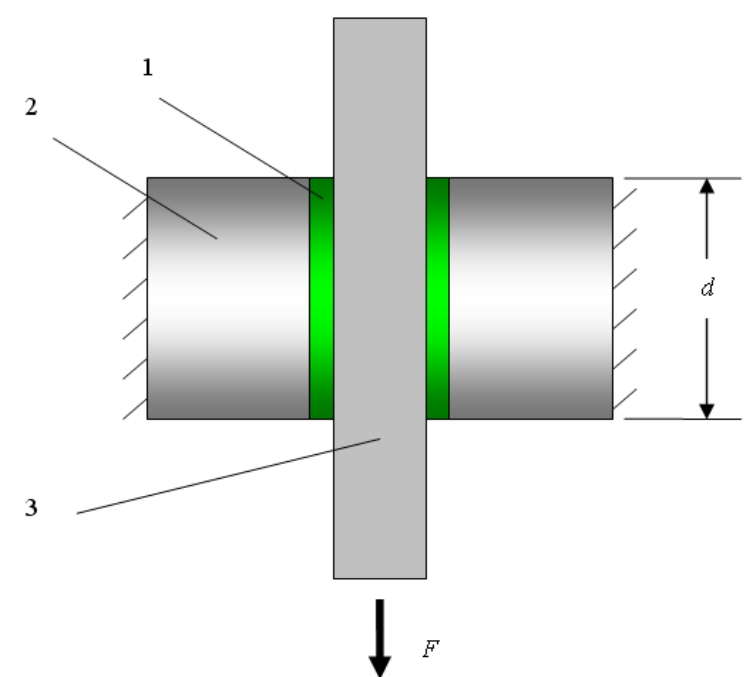

Fig. 8 yield stress measurement F: external force; $d$ : diameter of sealing area 1, Bingham plastic; 2, fixed Block 3 , movable plate

\section{Sealing Pressure}

Sealing pressure is dependant on sealing length, $\mathrm{L}$, sealing gap, $\mathrm{H}$, and yield stress of Bingham plastic. Sealing pressure with various sealing gaps and sealing lengths are listed in Table 2 and acceptable sealing dimensions for vacuum clamping are mark as blue. The curves of sealing pressure are plotted in Fig.9. From the results, it clearly highlights that the sealing length is large enough a good sealing capacity is achieved. The sealing gap thickness depends on the surface geometry tolerance, such as the milling tolerance $(0.1 \mathrm{~mm})$.

Table 2 Sealing pressure with various sealing gaps and lengths, $\mathrm{Pa}$

\begin{tabular}{crrrrrrrr}
\multicolumn{10}{c}{$\tau_{0}=626.8 \mathrm{~Pa}$} \\
\hline \multirow{2}{*}{$\mathrm{H}(\mathrm{m})$} & \multicolumn{1}{c}{0.01} & \multicolumn{1}{c}{$\mathrm{L}(\mathrm{m})$} & \multicolumn{1}{c}{0.03} & \multicolumn{1}{c}{0.04} & \multicolumn{1}{c}{0.05} & \multicolumn{1}{c}{0.06} & \multicolumn{1}{c}{0.07} & \multicolumn{1}{c}{0.08} \\
\hline 0.00005 & 250720 & 501440 & 752160 & 1002880 & 1253600 & 1504320 & 1755040 & 2005760 \\
0.0001 & 125360 & 250720 & 376080 & 501440 & 626800 & 752160 & 877520 & 1002880 \\
0.0002 & 62680 & 125360 & 188040 & 250720 & 313400 & 376080 & 438760 & 501440 \\
0.0003 & 41787 & 83573 & 125360 & 167147 & 208933 & 250720 & 292507 & 334293 \\
0.0004 & 31340 & 62680 & 94020 & 125360 & 156700 & 188040 & 219380 & 250720 \\
0.0005 & 25072 & 50144 & 75216 & 100288 & 125360 & 150432 & 175504 & 200576 \\
0.0006 & 20893 & 41787 & 62680 & 83573 & 104467 & 125360 & 146253 & 167147 \\
\hline
\end{tabular}




\section{Error Average Effect}

The surface roughness and waviness can be averaged by Bingham plastic because of its low yield stress and a well distributed pressure pattern will act on the component surface instead of a concentrated force, as shown in Fig.10 a. On the contrary traditional "O" ring sealing will result in a direct contact with a large concentrated force, as shown in Fig.10 b. The clamping distortion and location error will be greatly reduced because of the error average effect of Bingham sealing in a vacuum clamping comparing with "O" ring sealing.

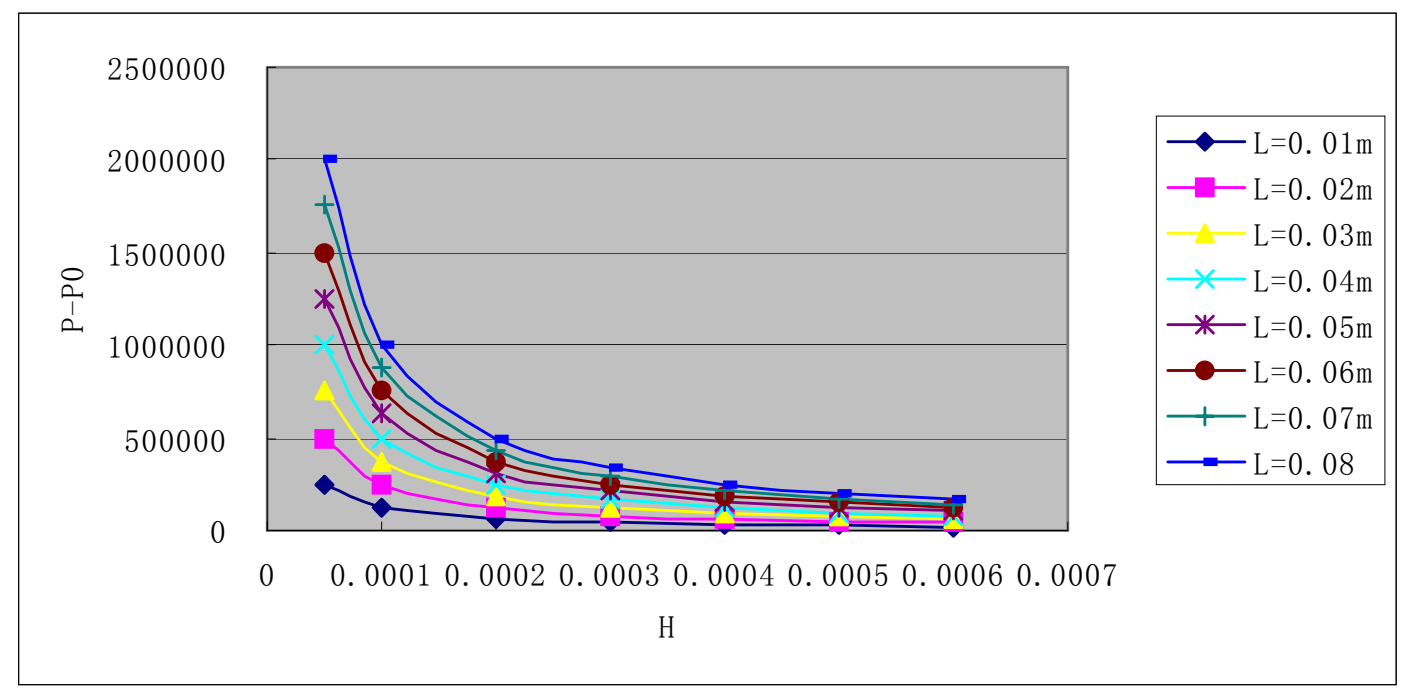

Fig.9 Curves of sealing pressure with developed Bingham plastic

L: sealing length; H: sealing gap thickness; P-P0: sealing pressure

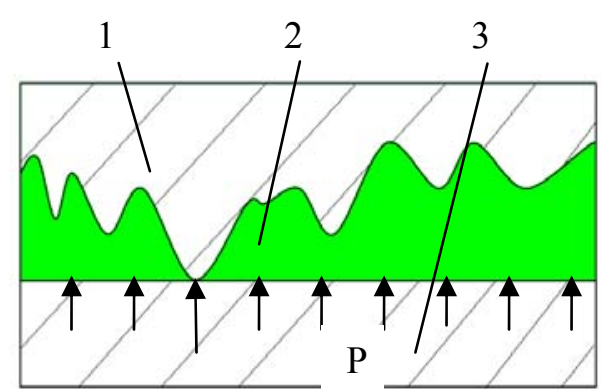

(a)

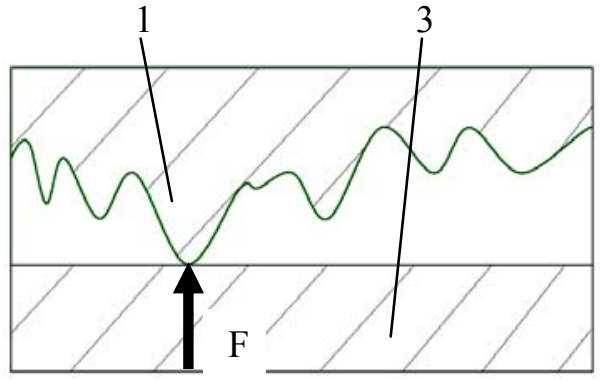

(b)

Fig.10 Error average function of Bingham sealing in a vacuum clamping

P: distributive pressure; F: concentrated force

1 component surface; 2 Bingham plastic; 3 clamping part

\section{Experimental Comparison}

Experimental comparison of the Bingham sealing and traditional "O" ring sealing is carried out in a blade fixture for the qualification of clamping distortion and vacuum value.

A clamping block with conforming surface is placed on the underside of a blade with an "O" ring groove is produced along the boundary of support area. A micrometer is placed in the middle of blade tip to measure the clamping distortion along axial direction, see Fig.11. The clamping distortion and vacuum value are listed in Table 3. When Bingham sealing is used instead of "O" ring, the distortion 
Trends in Aerospace Manufacturing 2009 International Conference

IOP Publishing

IOP Conf. Series: Materials Science and Engineering 26 (2011) 012021 doi:10.1088/1757-899X/26/1/012021

produced will be reduced to less than half level (42.5\%) and the vacuum values achieved stays constant.

Table 3 Experimental comparison for distortions of vacuum clamping

\begin{tabular}{cccccccc}
\multicolumn{8}{c}{ with Bingham sealing and "O" ring sealing } \\
\hline \multicolumn{2}{c}{ Test } & 1 & 2 & 3 & 4 & 5 & Average \\
\hline "O" ring & $\begin{array}{c}\text { Distortion } \\
(\mathrm{mm})\end{array}$ & 0.33 & 0.30 & 0.35 & 0.30 & 0.33 & 0.32 \\
$\mathrm{D}=6 \mathrm{~mm}$ & $\begin{array}{c}\text { Vacuum } \\
(\mathrm{Pa})\end{array}$ & 960 & 950 & 950 & 950 & 960 & 954 \\
$\begin{array}{c}\text { Distortion } \\
\text { Bingham } \\
\mathrm{L}=10 \mathrm{~mm})\end{array}$ & 0.10 & 0.15 & 0.13 & 0.15 & 0.15 & 0.136 \\
\hline $\begin{array}{c}\text { Vacuum } \\
(\mathrm{Pa})\end{array}$ & 950 & 960 & 950 & 950 & 950 & 952 \\
\hline
\end{tabular}

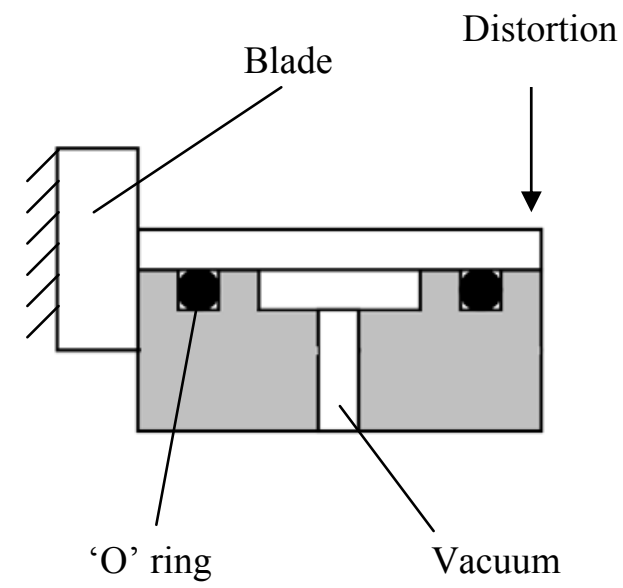

'O' ring sealing

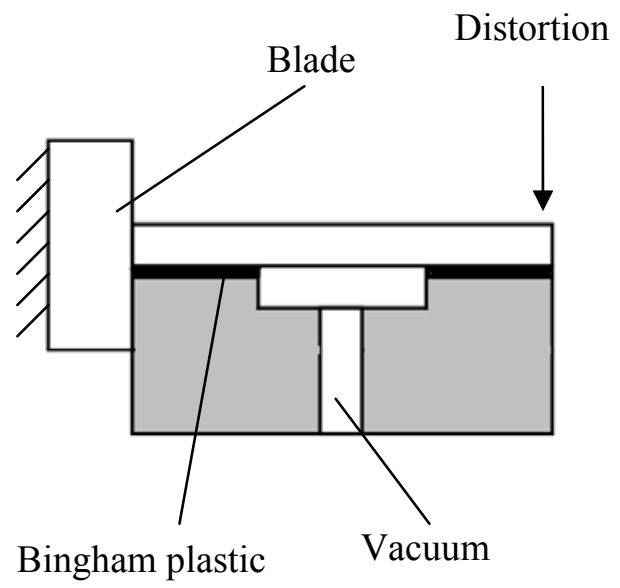

Bingham sealing

Fig.11 Experimental comparison of the Bingham sealing and traditional "O" ring sealing

\section{Conclusion}

From the above analysis and experimental comparison the conclusions can be drawn as the following.

1) Bingham plastic can be used as a good sealing medium which is easily applied on both static sealing and low speed rotational mechanism. The sealing mechanism will be sufficiently simplified. The yield stress can be measured with easy to evaluate the performance of Bingham plastic which will be used in application of Bingham sealing.

2) Bingham sealing capability is proportional to the ratio of sealing length, sealing gap thickness and the yield stress of the Bingham plastic. A high ratio of sealing length and sealing gap thickness is easily achieved up to 100 or 1000 in engineering cases. Therefore a high level sealing capability will be guaranteed by the low-yield-stress Bingham plastic. 
3) Vacuum clamping benefits from the use of Bingham sealing particularly: a) The location error reduced, b) the concentrated force avoided and it is a good protection for surfaces of components to be machined, c) a low level of clamping distortion (42.5\%) is witnessed comparing with the "O" ring sealing in experimental comparison of vacuum clamping.

Bingham sealing is particularly suitable for the vacuum clamping of a thin-walled component such as large and/or hollow blades because of the following advantages:

(1) No contact (or low level) stress and relevant deformation comparing with traditional "O" ring sealing in a vacuum clamping;

(2) No special requirements for surface finishing (roughness, flatness and roundness), adaptive fill-in for various geometric gap;

(3) Simplified mechanism. No additional mechanism (such as electrical loop, magnetic loop, and ' $\mathrm{O}$ ' ring groove) required except Bingham plastic with proper performance.

\section{Reference}

[1] W. Hoffmann. Clamping by Vacuum. Holzals Roh-und Werkstoff, 1992; 50(11):449-454.

[2] Anon. Vacuum Clamping Speeds Deburring. Manufacturing Engineering, 2004; 132(5):159-160.

[3] H Witte. Improved Machining Possibilities for Thin-walled Work-Pieces by Vacuum Clamping. Werkstattstechnik Zeitschrift Fur Industrielle Fertigung, 1986; 76(4): 223-225.

[4] Zunmin Geng. Fixture for Thin-Walled Shell/Cylindrical Components. WO2008107672, 2008.

[5] E.C. Bingham. An Investigation of the Laws of Plastic Flow. U.S. Bureau of Standards Bulletin, 1916; 13: 309-353.

[6] http://en.wikipedia.org/wiki/Bingham_plastic - cite_ref-2E. C. Bingham. Fluidity and Plasticity. McGraw Hill, New York, 1922; 219.

[7] RJ Atkin, TJ Corden, TG Kum and WA Bullough. 2-Dimensional Bingham Plastic Plow in a Cylindrical Pressurized Clutch. International Journal of Modern Physics, 1994; 8: 20-21.

[8] M.L. Wang and Y. Cheng. Magnetic-rheological sealing Roller. China Patent, 2004; ZL 200420109587.

[9] A. Fransson, CF Tsang, J Rutqvist, and G Gustafson. A New Parameter to Assess Hydromechanical Effects in Single-Hole Hydraulic Testing and Grouting. International Journal of Rock Mechanics and Mining Sciences, 2007; 44(7):1011-1021.

[10] Jiro Tsukahara, Kazumori Minami, Yoichi Hosoya. Gas Barrier Film and Method for Sealing Display and Device with Gas Barrier Film. US Patent, 2009; US2009/0061223 A1.

[11] Freyer Rune. A Method and a Device for Sealing a Void Incompletely Filled with a Cast Material. International Patent, 2005; WO 2006/065144A1. 